\title{
Use of Flat Ribbon like Electrode Geometry to Pole PVDF Piezoelectrics in Solution Processing
}

\author{
Prakash Kodali
}

\begin{abstract}
We study how ribbons of fluids subjected to electric fields can serve applications in energy harvesting. In particular the emphasis is on how the geometry (i.e. 2-D ribbons) can influence functionality. For applications related to energy harvesting, we consider the use of polymer Piezo-electric PolyvinylideneFluoride (PVDF). Corona poling, photo-induced, photo-thermal and electron beam poling are the different conventional techniques used for PVDF poling. The parallel plate capacitor structure made for poling the PVDF material while the $P V D F$ is being cured. One key advantage of preparing PVDF is the ability of solution processing. Normally, the liquid is then spin coated on a substrate and left to dry. Either during the process of spin coating, or after drying - the film of PVDF is poled so as to align the dipoles and make a piezoelectric. We propose the use of a metal-insulator ribbon like electrode geometry to combine the process of fabrication and poling thereby making the process more efficient. On the application of a voltage across the electrodes, the voltage of Vs is developed across the fluid. This result in a field of Vs/d across the PVDF fills aiding the process of poling while the film is in liquid phase. Therefore the ribbon like geometry aids the use of PVDF piezo-electrics in two ways. Firstly, it makes the fabrication process efficient by combining the poling with the structure development. Secondly, the control of width (w) and length (l) aids the setup of the $P V D F$ piezoelectric resonant frequency for a given thickness $(d)$. This helps match the resonant frequency of the ribbon with the incoming low frequency vibration to improve the energy harvesting levels. Piezo-electrics can be used in submerged applications, large area PVDF energy scavengers, mechanical filters and sensors, rural electrification, and charging circuits for hand-held devices.
\end{abstract}

Keywords: Piezo-electrets; Large Electronics; Energy Harvesting; Impedance Matching, Sensing and Resonance.

\section{INTRODUCTION}

When the structure of the familiar polythene - $(-\mathrm{CH} 2-$ $\mathrm{CH} 2-) \mathrm{n}$ - is changed by replacing the two hydrogen atoms with two fluorine atoms \{i.e., $-(-\mathrm{CH} 2-\mathrm{CF} 2-) \mathrm{n}$ - $\}$, it exhibits piezoelectricity due to the assymetricity. This polymer, known as polyvinyldene fluoride (PVDF) used in various applicatins. Since its discovery in 1969 [1], PVDF has gained immense popularity because of its versatility and wide-spread use in acoustics [2], [3], energy harvesting [1,4-7], biomedicine, micro-electro-mechanical systems (MEMS) and bio-MEMS applications [8, 9], vibration sensing [10, 11], pressure sensing [12 - 14], strain and deformation sensing $[15,16]$ slip sensing [17] and tactile sensing [13], [17 - 19] and vibration mode sensing [20, 21].PVDF has a relative dielectric coefficient of 11.5 that remains stable over a wide frequency range. It exhibited chemical inertness, continuous polarization and is easy to fabricate as large sheets with thickness between $9 \mu \mathrm{m}$ and 1

Revised Manuscript Received on December 02, 2019

* Correspondence Author

Prakash Kodali*, Assistant Professor, E.C.E Department, National Institute of Technology, Warangal, India. Email: kprakash@nitw.ac.in $\mathrm{mm}$. Its acoustic impedance close to that of water with a flat response curve, which makes suitable for sonar devices. It has a piezoelectric coefficient $\mathrm{d}_{33} \approx 15 \mathrm{nC} / \mathrm{N}$ and $\mathrm{d}_{31}=\mathrm{d}_{32} \approx 6$ $\mathrm{nC} / \mathrm{N}$. It has a Young's modulus of about $2500 \mathrm{MPa}$. The melting point of PVDF is about $170^{\circ} \mathrm{C}$.

Piezoelectric energy harvesting and sensing applications are largely at the development stage and also in the developing stage, although some devices are commercially available to use in various applications [22-24]. There are many claims in scientific literature about them efficiency and expected performance in all space and ground use. In this paper we discuss the use of the metal-insulator-channel architecture used for the purpose of desalination with renewed applications to the confinement and field effect based poling of PVDF. This makes a novel leap in achieving the patterning of any desired 2D geometry alongside poling at high voltages. The poled ribbon of PVDF would then retain geometrical advantages that enhance its functionality for energy harvesting.

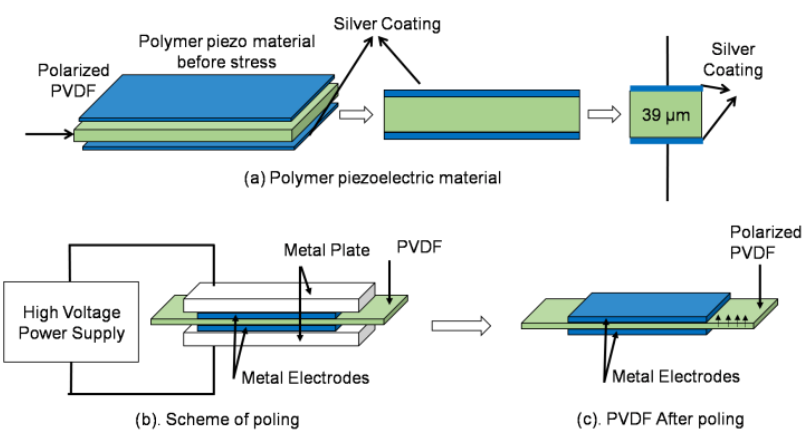

Fig. 1. Polymer material and poling (a). Polymer Piezo electric material structure. (b). Poling of a PVDF in a conventional mode, Polarization of polymer ribbon by high voltage direct current device. (c). Polarized PVDF after applying high voltage for long time.

\section{CONVENTIONAL METHODS TO POLARIZE PVDF}

\section{A. PVDF Solution Process and Film Development}

Of many techniques, one of the methods of preparing PVDF is as follows. PVDF powder $1 \mathrm{mg}$ is dissolved in mixed solution of $5.6 \mathrm{ml}$ of 1 - methyl -2 - pyrollidone and $0.4 \mathrm{ml}$ of methyl - ethyl - ketone. The mixture is kept at $65^{\circ} \mathrm{C}$ and stirred at $200 \mathrm{rpm}$ for $15 \mathrm{~min}$. The stirring is then enhanced to $100 \mathrm{rpm}$ at $80^{\circ} \mathrm{C}$. Finally, the viscous solution is filtered to remove particulates to ensure a homogeneous solution of PVDF. This solution is then spin coated on a smooth substrate to the desired thickness and subsequently baked at $70^{\circ} \mathrm{C}$ for 1 hour. 


\section{Use of Flat Ribbon like Electrode Geometry to Pole PVDF Piezoelectrics in Solution Processing}

The PVDF film is finally peeled away from the substrate thereby resulting in a free standing film of PVDF. To establish electrical contacts, silver $(\mathrm{Ag})$ or aluminium $(\mathrm{Al})$ is thermally evaporated onto the two sides of the PVDF sheet. The structure of the PVDF ribbon is as shown in Figure 1. Either prior to or post metallization, it is essential that the PVDF material be poled. This rather important process is discussed in the subsequent section.

\section{B.Poling of PVDF}

Over the years there has been a lot of wisdom and contradictions developed over what constitutes the best poling method. It is believed that poling of PVDF is enhanced at higher temperatures where the molecules have greater energy to move and align. However, techniques such as corona poling have shown this rather logical argument to be a myth. The second belief was that the ideal poling time was about 30 min. However, this too has been contradicted when a continued increase in poling activities was observed over an increase in time. There has also been a notion of stretching the PVDF prior to poling thereby permitting a more uniform electric field through the material. Other studies have shown that simple stretching without the presence of an electric field is itself sufficient to pole the PVDF. It is concluded that the stretching transforms a lot of $\alpha_{p}$ - phase to $\beta_{p}$ - phase. The most useful phase in the PVDF electret is the $\beta_{p}$ - phase, which can be achieved by mechanical stretching or electrical poling [25]. The $\alpha_{p}$ - phase may not be influenced i.e. show piezoelectricity by the stretching of PVDF film. This reorientation of the molecule to enhance piezoelectricity is accomplished by the process of poling. Typical poling fields are $700 \mathrm{kV} / \mathrm{cm}$.

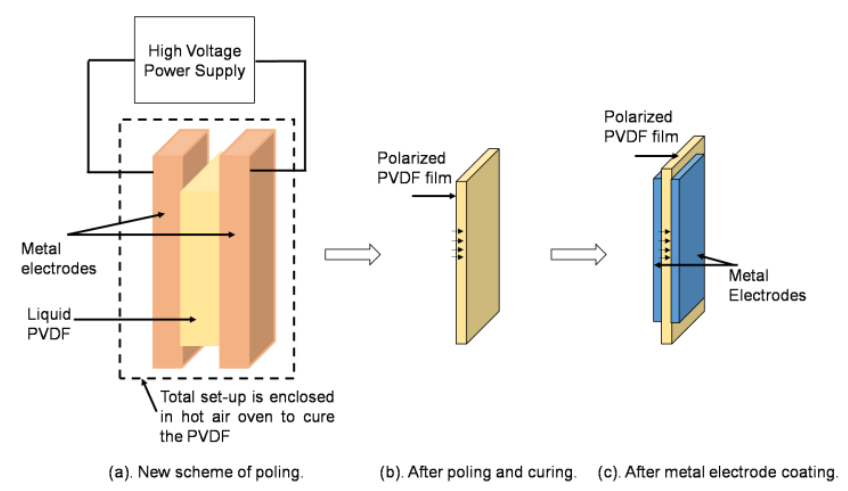

Fig. 2. PVDF making and poling process. (a). The scheme of PVDF poling with liquid PVDF material filled in channel. (b). Polarized PVDF after curing. (c). Poled PVDF film with metal electrode.

There are many techniques to achieve poling. Direct poling, Corona poling and Photo-induced are the most common methods. Direct poling is used after metallization and is shown in Figure 1(b). In this technique, a large voltage is applied across the metal electrodes thereby resulting in an electric field through the PVDF. The application of high voltages dramatically boosts poling efficiency since the high voltage not only establishes a large electric field, but also amplifies this electric field due to the compression of the PVDF film by electrostatic forces seen between the plates. However, the presence of a large voltage usually results in arcing between the electrodes or the breakdown of the PVDF film and physical damage due to the establishments of large currents. In order to limit the currents, Corona poling is used. In this technique the PVDF is metalized on only one side or sometimes not even that.

The active electrode is separated from the PVDF film by an air gap. This permits the use of a high voltage while not risking the flow of high currents into the PVDF. However, the breakdown of air would result in negating this purpose. To prevent this, the entire setup is placed in vacuum, or the air gap was replaced with paraffin or silicone oil. In order to improve the electric field intensity, the active electrode geometry is made to have a high curvature. Therefore electrodes such as a wire achieve large area poling while at the same time maintaining high electric field intensity at lower voltages. In photo-thermal and photo-induced poling, glass coated with indium tin-oxide a transparent electrical conducted is brought in contact with the PVDF. High intensity, coherent light produced by laser beam irradiates the PVDF through the ITO coated glass. The electric fields generated by the electro-magnetic waves are used to achieve poling. Any polarized electromagnetic wave could be used to achieve this purpose.

\section{EXPERIMENTAL SETUP}

Proposed one is entirely different from conventional poling methods as when the PVDF film is sandwiched between metal plates or ITO-coated glass sheets. Novel Integrated Fabrication explained below.

System Design and Poling - In our approach, distinct from conventional methods, liquid PVDF is used and placed between the electrodes or it made to flow between the electrode plates. The overall structure consists of a metal-liquid PVDF-metal format shown in Figure 2 (a). To generate different geometries of PVDF, the electrodes of the system were based on aluminum sheets that were patterned using a $\mathrm{CO}_{2}$ laser tool instrument. The pattern design was made in CAD (Solid Works 2012) which is shown in Figure 3 (b). Then the metal cut pieces were arranged in a manner that the complete arrangement permitted the creation of the channel. The top and bottom of the structure was covered with tape. Using comb structure poling method allows higher electric field applied in poling and able to get more electricity and sensitivity in various applications.
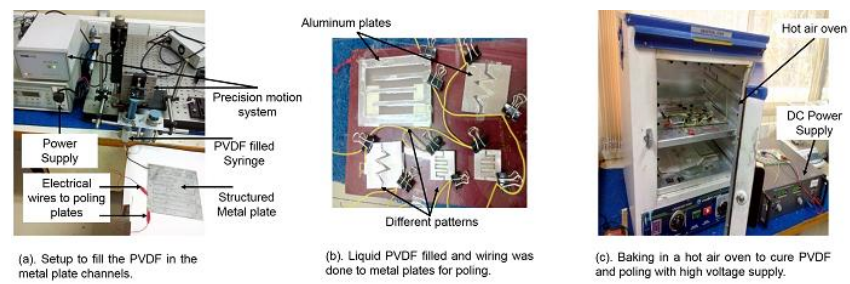

Fig. 3. Poling system setup (a). Injecting liquid PVDF to a parallel plate system using syringe. (b). Different structures of PVDF materials are made using these aluminum patterns. Liquid form PVDF is filled in the gaps. (c). Curing and poling set up for different structures making of PVDF for sensing and energy harvesting by electric responses. 
The prepared PVDF solution was injected into the channel using a controlled syringe pump. The flow now only permits confinement of the PVDF thereby shaping the geometry, but also mobilizes the PVDF molecules to preferentially orient themselves based on their surface interaction with the metal electrodes. Once the channel was filled with PVDF material, the top layer of tape was removed. To avoid leakages from the inlet and outlet, stoppers were used. The electrical contact were then wired to the top and bottom metal electrodes. The total setup is then placed in a hot air oven to cure the PVDF while simultaneously applying an electric field. The curing temperature was set at $700^{\circ} \mathrm{C}$ for 1 hour while the poling lasted was 2 hours at a DC voltage of $200 \mathrm{~V}$. Such a technique permits a significant saving of energy. Conventionally, the PVDF is cured, and then reheated to pole. However, in this technique the liquid PVDF is baked only once and is poled simultaneously. The entire setup is shown in Figure 3(c). Once the PVDF is poled and cured simultaneously, the film is disengaged from the system and the top and bottom surfaces of the PVDF film are coated with Ag or Au electrodes using thermal evaporation as shown in Figure 4 (a) and (b). (a). After poling and curing liquid PVDF.

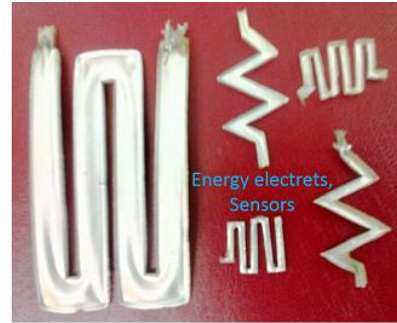

(b). After metal(Al) electrode coating.
Fig. 4. PVDF materials after curing in hot air chamber. (a). PVDF was poled and cured simultaneously in a hot oven with high voltage DC supply. (b). after poling and curing different structures of PVDF materials, top and bottom layers are coated with the aluminum metal using a thermal evaporation system.

\section{GEOMETRY AND FUNCTIONALITY}

With regards to energy harvesting, different geometries can have significant impact on performance. For example, consider three geometries. The first is a simple $2 \mathrm{D}$ ribbon with $\mathrm{d}<<\mathrm{w}<<1$. The second is a 3D rope, with $\mathrm{d} \sim \mathrm{w}<<1$. The final is a $2 \mathrm{D}$ serpentine ribbon with $\mathrm{d}<<\mathrm{w}<<1$. These structures are shown in Figure 3 and Figure 4. In the case of a simple 2D ribbon, the natural frequency of the ribbon is easily guessed by control of the tension in the ribbon and the length/width of the ribbon. If the width of the ribbon is kept much smaller than the length, modes of vibration of the ribbon are very confined with the ribbon tending to behave as a string. When the natural frequency of the ribbon is known, the piezoelectric ribbon can be shaped to maximize vibration and hence charge production at that frequency can be more. Moreover, the ribbon permits significant stress due to the low thickness (low d). Therefore the ribbon geometry is more apt to be used at low force regimes where the mechanical stress does not exceed the yield stress of PVDF.

\section{Characterization and electrical response of proposed sample}

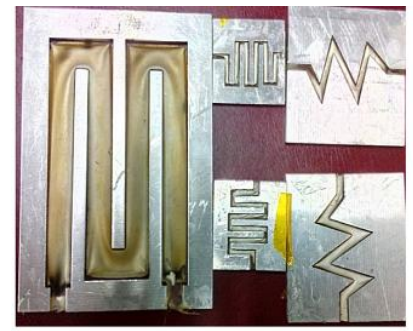

Degree of the crystallinity is the important factor in PVDF sample investigation, Differential Scanning Calorimeter and Fourier Transformed Infrared Spectroscopy are used to measure the $\beta_{p}$ - phase. The degree of crystallinity $\left(X_{c}\right)$ with differential scanning calorimeter is calculated with set temperature range [26].

$$
X_{c}=\frac{\Delta H_{f e}}{\Delta H_{f e}^{*}}
$$

Where $X_{c}, H_{f e}$ are degree of crystallinity, fusion enthalpy of the sample PVDF and $H *_{f e}$ is fusion enthalpy with $100 \%$ crystallinity, which is $102.5 \mathrm{~J} / \mathrm{g}$ given in [27]. Fraction of $\beta_{p}$-phase crystals $F\left(\beta_{p}\right)$ in each sample is calculated by:

$$
F\left(\beta_{p}\right)=\frac{A_{\beta p}}{\left(\frac{k_{\beta p}}{k_{\alpha_{p}}}\right) A_{\alpha_{p}}+A_{\beta p}}
$$

Where $A \alpha_{p}$ and $A_{\beta p}$ is determined by absorption bands for $\alpha_{p}$ - and $\beta_{p}$-phases of proposed sample and can be estimated by product term

$$
A_{\alpha_{p}, \beta_{p}}=C_{p} \cdot K_{\alpha_{p}, \beta_{p}} \cdot X_{\alpha_{p}, \beta_{p}} \cdot t_{p}
$$

Where $C_{p}$ is monomer concentration, $\alpha_{p}$ and $\beta_{p}$ subscripts refer to the crystalline phases. $K$ is the absorption coefficient at the respective wave number, and $X_{c}$ is degree of crystallinity defined in above equation, $t_{p}$ is thickness of PVDF film.

For the PVDF materials or samples d31, d32 and d33 are piezoelectric charge constants, which are characterized by d31 meter respectively. Two pieces of polyethylene HDPE plastic are in PVDF sample. Generally pressed and cantilever structures are used and we proposed a long spring structures shown in figures 5 (b), 6 (a) and (b). Different stretched PVDFs, force applied and measured quantity output voltages are shown in figure 6 (a) and (b).

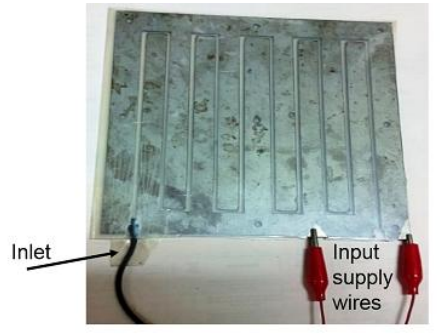

(a). Metal structure used for long PVDF thread making.

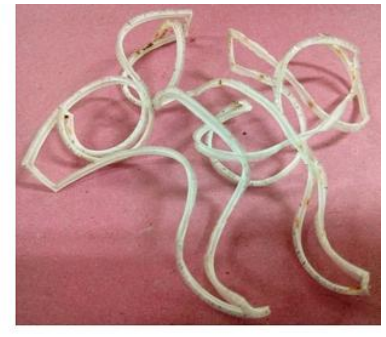

(b). Poled long PVDF thread.
Fig. 5. Getting a long PVDF threads in new scheme of poling. (a). Metal pattern used to get the long thread. (b). Long PVDF thread is made using a comb structure of electrode placement and it was poled while curing.

In the case of the $3 \mathrm{D}$ rope, the stress profile is made much more being due to the large $\mathrm{d}$. Therefore the $3 \mathrm{D}$ rope permits applications in areas where the force profile is much wider. Nevertheless, the rope permits a good definition of the natural frequency. The $3 \mathrm{D}$ rope has more aspects of mechanical excitation. Since the mass density of the 3D rope is larger than that of the ribbon,

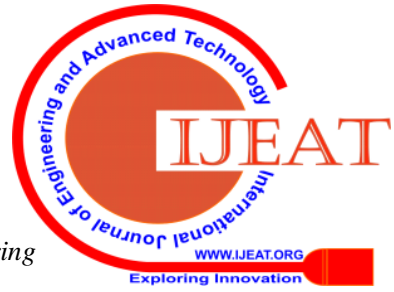


any angular velocity would result in a good centrifugal force sufficient to excite a significant voltage across the piezoelectric rope. Moreover due to large $\mathrm{d}$ both all modes $\mathrm{d} 31, \mathrm{~d} 32, \mathrm{~d} 21$ can be tapped into and sensed. This is unlike the case of a ribbon where it is difficult to pattern electrodes along the thickness. The case of the meandered 2D ribbon also provides interesting possibilities. Since any small tensile force results in very large stress at the concave rounded edges of the meanders, this setup could be used to measure very small forces. The integrated use of such geometries along one integrated system opens up interesting applications.

\section{RESPONSE OFELECTRETS}

The serpentine structure of PVDF is made with the proposed poling technique and an aluminum metal film is formed. The response was taken, after stretching along the two ends points of the PVDF structure. In Figure 6(a) the response of the PVDF was captured in CRO. This structure is different from the regular straight strip, and its response is also very efficient. The response was taken when the strip was stretched. It is noticed that the open circuit voltage is about $200 \mathrm{mV}$. Another strip was made wider and longer, was $6 \mathrm{~mm}$ wide, $50 \mu \mathrm{m}$ thick and $250 \mathrm{~mm}$ long. This PVDF film was fixed to rigid supports with electrical tapping to take the response. The electrical response is shown in Figure 6(b). When an indirect mechanical input was given to the PVDF film, that response had a high electrical output $1 \mathrm{~V} p$-p compared to the conventional poled film. The response is good and the occupied area of the film is less compared to a long PVDF film.

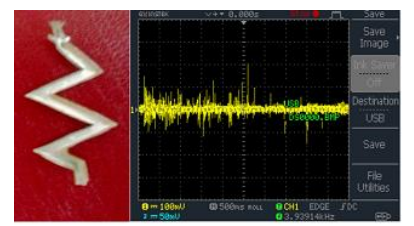

(a). Structure different from regular strip of
a PVDF and its response when stretched.

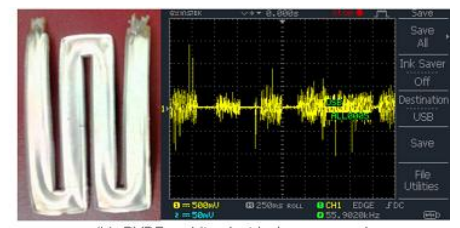

(b). PVDF and its electrical response when
it got excited by the mechanical input.
Fig. 6. Electrical responses of poled PVDF. (a). Stretching and releasing of $P V D F$ is giving an electrical response. (b). Electrical response of a PVDF stretched in between rigid poles and tapped at low frequency i.e., giving an indirect mechanical input.

\section{CONCLUSION}

This paper introduced a novel fabrication and patterning technique for PVDF using the same metal-insulator-channel setup used for several applications. The novelty in the fabrication allowed for the PVDF to be patterned into any geometry while permitting poling simultaneously. This avoids energy and process time spent in baking, stretching, subsequent poling and then patterning to an all integrated one step solution processed fabrication flow path. This is the first novel contribution with regards to energy harvesting. The second novel contribution with regards to energy harvesting with the 2D ribbon generated by field effect on a fluid (PVDF solution). The ribbon provides a useful benefit of fixing the resonant frequency easily while permitting energy scavenging with low frequency vibrations. This aspect is dealt with in greater details using experiments and models of

the poled PVDF films act as a good energy generator. For 2 mg of weight of sample, 30-40 $\mu \mathrm{A}$ current and $200-500 \mathrm{mV}$ voltages are noticed.

\section{REFERENCES}

1. H. Kawai, "The piezoelectricity of poly (vinylidene fluoride)," Japanese Journal of Applied Physics, vol. 8, 1969, pp. 975.

2. J. Xu, M. Dapino, D. Gallego-Perez, and D. Hansford, "Microphone based on polyvinylidene fluoride (PVDF) micro-pillars and patterned electrodes," Sensors and Actuators A: Physical, vol. 153, 2009, pp. 24-32.

3. B.-T. Wang, "The PVDF-based wave number domain sensing techniques for active sound radiation control from a simply supported beam," The Journal of the Acoustical Society of America, vol. 103, 1998, pp. 1904-1915.

4. Q. Zhang, V. Bharti, and G. Kavarnos, "Poly (vinylidene fluoride) (PVDF) and its copolymers," Encyclopedia of Smart Materials, 2002.

5. D. Reneker, J. Gorse, D. Lolla, C. Kisielowski, J. Miao, P. Taylor, et al., "Polyvinylidene fluoride molecules in nanofibers, imaged at atomic scale by aberration corrected electron microscopy," Bulletin of the American Physical Society, 2016.

6. K. Omote, H. Ohigashi, and K. Koga, "Temperature dependence of elastic, dielectric, and piezoelectric properties of "single crystalline" films of vinylidene fluoride trifluoroethylene copolymer," Journal of applied physics, vol. 81,1997, pp. 2760-2769.

7. K. Prevedouros, I. T. Cousins, R. C. Buck, and S. H. Korzeniowski, "Sources, fate and transport of perfluorocarboxylates," Environmental science \& technology, vol. 40, 2006, pp. 32-44.

8. C.-H. Hung, Y.-L. Lin, and T.-H. Young, "The effect of chitosan and PVDF substrates on the behavior of embryonic rat cerebral cortical stem cells," Biomaterials, vol. 27, 2006, pp. 4461-4469.

9. D. Gallego-Perez, N. J. Ferrell, N. Higuita-Castro, and D. J. Hansford, "Versatile methods for the fabrication of polyvinylidene fluoride microstructures," Biomedical microdevices, vol. 12, 2010, pp. 1009-1017.

10. M. W. Shafer, M. Bryant, and E. Garcia, "Designing maximum power output into piezoelectric energy harvesters," Smart materials and structures, vol. 21, 2012, pp. 085008.

11. F. Wang, M. Tanaka, and S. Chonan, "Development of a PVDF piezopolymer sensor for unconstrained in-sleep cardiorespiratory monitoring," Journal of intelligent material systems and structures, vol. 14, 2003, pp. 185-190.

12. I. Lee and H. Sung, "Development of an array of pressure sensors with PVDF film," Experiments in Fluids, vol. 26, 1999, pp. 27-35.

13. B. P. Mahale, D. Bodas, and S. Gangal, "Development of PVdF based pressure sensor for low pressure application," in Nano/Micro Engineered and Molecular Systems (NEMS), 2011 IEEE International Conference on, 2011, pp. 658-661.

14. T. Sharma, S.-S. Je, B. Gill, and J. X. Zhang, "Patterning piezoelectric thin film PVDF-TrFE based pressure sensor for catheter application," Sensors and Actuators A: Physical, vol. 177, 2012, pp. 87-92.

15. Y.-J. Hsu, Z. Jia, and I. Kymissis, "A locally amplified strain sensor based on a piezoelectric polymer and organic field-effect transistors," Electron Devices, IEEE Transactions on, vol. 58, 2011, pp. 910-917.

16. J. Yi and H. Liang, "A PVDF-based deformation and motion sensor: Modeling and experiments," Sensors Journal, IEEE, vol. 8, 2008, pp. 384-391.

17. B. Choi, H. R. Choi, and S. Kang, "Development of tactile sensor for detecting contact force and slip," in Intelligent Robots and Systems, 2005.(IROS 2005). 2005 IEEE/RSJ International Conference on, 2005, pp. 2638-2643.

18. Y. Yamada, H. Morita, and Y. Umetani, "Vibrotactile sensor generating impulsive signals for distinguishing only slipping states," in Intelligent Robots and Systems, 1999. IROS'99. Proceedings. 1999 IEEE/RSJ International Conference on, 1999, pp. 844-850.

19. S. Sokhanvar, M. Packirisamy, and J. Dargahi, "A multifunctional PVDF-based tactile sensor for minimally invasive surgery," Smart materials and structures, vol. 16, 2007, pp. 989.

20. C.-K. Lee and F. C. Moon, "Modal sensors/actuators," Journal of applied mechanics, vol. 57, 1990, pp. 434-441.

21. H. Sumali, K. Meissner, and H. H. Cudney, "A piezoelectric array for sensing vibration modal coordinates," Sensors and Actuators A: Physical, vol. 93, 2001, pp. 123-131. 
22. T. Bailey and J. Ubbard, "Distributed piezoelectric-polymer active vibration control of a cantilever beam," Journal of Guidance, Control, and Dynamics, vol. 8, 1985, pp. 605-611.

23. P. Ueberschlag, "PVDF piezoelectric polymer," Sensor Review, vol. 21, 2001, pp. 118-126.

24. M. Tamura, T. Yamaguchi, T. Oyaba, and T. Yoshimi, "Electroacoustic transducers with piezoelectric high polymer films," Journal of the Audio Engineering Society, vol. 23, 1975, pp. 21-26.

25. V. Sencadas, M. V. Moreira, S. Lanceros-Méndez, A. S. Pouzada, and R. GregórioFilho, " $\alpha$-to $\beta$ Transformation on PVDF films obtained by uniaxial stretch," in Materials science forum, 2006, pp. 872-876.

26. X. J. Zhao, J. Cheng, S. J. Chen, J. Zhang, and X. L. Wang, Controlled Crystallization of Poly (vinylidene fluoride) Chains from Mixed Solvents Composed of Its Good Solvent and Nonsolvent. J. Polym. Sci. B. 48, 2010, pp. 575-581.

27. W. T. Mead, A. E. Zacharidas, T. Shimada, and R. S. Porter, Solid State Extrusion of Poly (vinylidene fluoride). 1. Ram and Hydrostatic Extrusion. Macromolecules. 12, 1979, pp. 473-478.

\section{AUTHOR PROFILE}

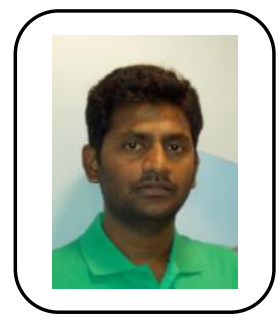

Prakash Kodali completed Bachelor of Technology in the stream of Electronics and Communication Engineering from JNTU affiliated NEC. After Under Graduation program, he received the Master of Technology (M.Tech) degree In Department of Electronic Systems Engineering (DESE) - (Formerly CEDT) from the Indian Institute of Science (IISc), Bangalore, India, 2010. He completed Ph.D in Instrumentation and Applied Physics Department from the Indian Institute of Science (IISc), Bangalore, 2016. Since 2011 he is working in the field area of Biomedical Electronics, Embedded Systems, Desalination, Energy Harvesting and Large Area Flexible Electronics product development. For the support of Bio - Warfare and Space applications he developed systems in the group of DRDO, ISRO and DST funded projects. 